\title{
Comparative Efficacy of Open-chain Kinematics and Closed-chain Kinematics on Walking Proficiency and Societal Integration in Post-stroke Individuals: a Pretest- Post-test Experimental Study
}

Obianuju Nwaedozie

University of Lagos

Caleb Gbiri

University of Lagos

Olajide Olawale

University of Lagos

Ukamaka Mgbeojedo ( $\sim$ amakamgbeojedo@yahoo.com )

University of Nigeria - Enugu Campus https://orcid.org/0000-0002-8090-7803

Caleb Adeagbo

University of Lagos

\section{Research Article}

Keywords: Open-Chain Kinematics, Closed-Chain Kinematics, Walking Proficiency, Societal Integration, Post-Stroke Individuals

Posted Date: November 11th, 2021

DOI: https://doi.org/10.21203/rs.3.rs-990536/v1

License: (c) (i) This work is licensed under a Creative Commons Attribution 4.0 International License.

Read Full License 


\section{Abstract \\ Background}

Post-stroke hemiplegic gait is a mixture of deviations and compensatory motion dictated by residual function. To improve stroke survivors' walking ability, it is necessary to evaluate different rehabilitation approaches and identify those that have a greater effect on locomotor recovery of stroke patients.

\section{Objectives}

This study was designed to compare the effect of open-chain kinematics (bicycle ergometry) and closedchain kinematics (treadmill) on walking proficiency in post-stroke individuals and their societal integration.

\section{Methods}

This was a pretest- posttest- experimental study involving 35 ambulatory hemiplegic stroke survivors (18males and 17 females) with a mean age of $53.77 \pm 10.95$ undergoing rehabilitation at the two Teaching Hospitals in Lagos. Patients went through a 10-week rehabilitation and were randomly assigned to two intervention groups. Spatio-temporal gait parameters were measured by the six-metre walkway and community integrated questionnaire was used to examine home integration, social integration and productive activities. Data were subjected to inferential and descriptive statistics. The level of significance was set at $p<0.05$.

\section{Results}

Results showed significant difference between baseline and post intervention scores for all the gait parameters in the bicycle ergometer group except for stride length $(p=0.078)$. There was also a significant difference in the mean change in cadence between the treadmill and bicycle ergometer group $(p=0.04)$.

\section{Conclusions}

Both open-chain and closed-chain kinematics are effective, but closed- chain is most effective in reeducating ambulation and re-gaining spatio-temporal gait parameters after stroke and should be structured into the patients' treatment regimen to effectively improve functional capability in post-stroke individuals.

\section{Plain English Summary}


Stroke is the leading cause of long-term disability, and its burden increases worldwide. Recovery from stroke is often slow and incomplete, leading to partial or complete loss of movement. The ability to walk independently is a prerequisite for the performance of most daily activities. In a post stroke hemiplegic gait, there is decreased walking speed, short or uneven step and stride length, increased stride width, increased double support, and dependence on support through the hands. Hence, the restoration of gait in stroke survivors became a major goal in rehabilitation with intervention aimed at optimizing walking performance

Treadmill training has been recently introduced as a measure to improve the post stroke gait pattern, but there is still limited data on the use of the bicycle ergometer. This study was intended to compare the effect of bicycle ergometry and treadmill on walking proficiency and societal integration in post-stroke individuals. Findings from this study reveal that both bicycle ergometry and treadmill are effective, but treadmill is most effective in re-educating walking after stroke and should be structured into the patients' treatment regimen to effectively improve functional capability in post-stroke individuals.

\section{Introduction}

Stroke is one of the principle causes of morbidity and mortality in adults worldwide and it is known to be a neurological deficit attributed to an acute, focal or global injury of the central nervous system by a vascular cause [1]. It is the leading cause of long-term disability, and its burden increases across the globe $[2,3]$. Recovery from stroke is often slow and incomplete, leading to partial or complete loss of locomotion, activities of daily living, cognition and communicative skills. The devastating stroke consequences have enormous personal, social and economic impact on oneself and the society at large [4].

Impairments resulting from stroke often lead to persistent difficulties with walking. Walking is an important human activity which enables us to be productive and participative members of a community [5]. Walking ability has important health implications in providing protective effects against secondary complications common after stroke. Poor walking ability has been found to reduce quality of life with a reduction in participation in activities outside the home and consequent social isolation [6]. The ability to walk independently is a prerequisite for the performance of most daily activities [7].

The capacity to walk in a community setting requires the ability to walk at speeds that enable an individual to cross the street in the time allotted by pedestrian lights, to step on and off a moving walkway, in and out of automatic doors, walk around furniture, under and over objects.

Stroke patients fear dependency more than anything else after a stroke; and the ability to walk independently has been found to provide the greatest protection against dependency [8]. Walking speed of community-dwelling people after stroke has been reported to be around $0.5 \mathrm{~m} / \mathrm{s}$ with studies reporting a range between 0.3 and $0.8 \mathrm{~m} / \mathrm{s}$ [9]. Walking capacity has also been found to be markedly reduced with 6-minute distance being reported as around $250 \mathrm{~m}$ ranging between 40 and $400 \mathrm{~m}$ [10]. Post-stroke hemiplegic gait is a mixture of deviations and compensatory motion dictated by residual function [11]. In 
a post stroke hemiplegic gait, there is decreased walking speed, short or uneven step and stride length, increased stride width, increased double support, and dependence on support through the hands [12]. Hence, the restoration of gait in stroke survivors became a major goal in rehabilitation with intervention aimed at optimizing walking performance by preventing adaptive changes in lower limb soft tissue, eliciting voluntary activation in key muscles groups in lower limbs, increasing muscle strength and coordination, increasing walking velocity and endurance, increasing flexibility and increasing cardiovascular fitness [13]. The major aim of stroke rehabilitation is to reduce disabilities and enable patient to return to society. It has been reported that only $7 \%$ of patients discharged from rehabilitation met the criteria for community walking which included the ability to walk $500 \mathrm{~m}$ continuously at a speed that would enable them to cross a road safely [14].

Therefore, it became necessary to evaluate different rehabilitation approaches and identify those that have a greater effect on locomotor recovery of stroke patient for faster societal integration. Recently, treadmill training has been introduced as a measure to improve the post stroke gait pattern [15], but there is still limited data on the use of the bicycle ergometer. Hence, this study was designed to compare the effect of open-chain kinematics and closed-chain kinematics on walking proficiency and societal integration in post-stroke individuals.

\section{Methods}

This was a pretest- post-test experimental study involving a total of 38 ambulatory hemiplegic stroke survivors (21 males and 17 females), out of which only 35 (18 males and 17 females) completed the study, giving an attrition rate of $92.1 \%$. Ethical approval for this study was obtained from the Health Research Ethics Committee of Lagos University Teaching Hospital (LUTH) Idi Araba, Lagos and Lagos State University Teaching Hospital (LASUTH) Ikeja, Lagos, Nigeria, where this research was carried out. Thirty five volunteering stroke survivors who were undergoing rehabilitation at the Physiotherapy Outpatient Department of the two tertiary hospitals were purposively recruited for this study. Participants were recruited if they met the inclusion criteria: were older than 18 years and whose diagnoses were confirmed by means of a CT scan and or an MRI, were able to understand verbal instructions and follow commands, were able to walk 6 metres independently with or without a walking aid. They were excluded if they have had previous stroke or brain injury. Informed consent was obtained from the participants before the commencement of the study. The participants' clinical data was obtained from their case note.

\section{Outcome measures}

The outcome measures used were the 6-meter walkway and a questionnaire for societal integration.

\section{Stopwatch (Leap Stop watch FXIT, China)}

A handheld stopwatch that counts to microseconds of time 


\section{Bicycle Ergometer (RUN-700/T, Italy)}

It is a device with saddle, pedals and handlebars arranged as on a bicycle but used as exercise equipment instead of transportation. It is also called stationary bicycle or exercise bicycle. It has low impact, does not put much stress on joints, it is safe and effective for cardiovascular exercise.

\section{Treadmill (Woodway 4Front treadmill, Germany)}

It is an exercise machine for running or walking in a place with a wide conveyor belt driven by an electric power or flywheel. The rate at which the belt moves is the rate of running or walking.

\section{6-Meter Walkway:}

This is a measure that was used to assess the quality of walking of the post stroke subjects. Subjects were be asked to walk a short distance ( 6 meters) at their usual pace and maximum speed and gait parameters (step length, stride length, speed and cadence) were measured.

\section{The Community Integrated Questionnaire (CIQ)}

This is a 15 item self-report questionnaire with 3 subscales which examine home integration, social integration and productive activities. School and volunteer activities are scored on a 6-point scale and the other 12-items are scored on a 3-point scale [16]. The three subscales are summed to yield a total score for community integration ranging from $0-29$, with higher scores indicating a greater degree of community integration.

\section{Procedures for Data Collection}

Participants were assessed at baseline using the 6-meter walkway and a questionnaire for societal integration. All participants were instructed to walk on a 6-meter walkway with sprinkled powder to show the footprints. The prints were utilized to measure the step length, stride length, speed and cadence.

The physiotherapy treatment sessions were carried out two times a week for 10 weeks at the end of which the gait parameters and Community Integration Questionnaire were administered again in order to obtain the post-treatment values. 


\section{Treatment procedure}

All participants were instructed to walk on a 6-meter walkway with sprinkled powder to show the footprints. Participants were instructed to walk $2 \mathrm{~m}$ away from the walkway, such that a uniform step would have been established before getting to the walkway. The prints were utilized to measure the step length, stride width, speed and cadence. The step length $(\mathrm{cm})$ was obtained by measuring the linear distance between two successive heel strikes (from affected limb to unaffected limb). The stride length $(\mathrm{cm})$ was obtained by measuring the linear distance from one heel strike of the affected limb to the next heel strike of the same limb. The natural gait speed (walking velocity) $(\mathrm{m} / \mathrm{s})$ was determined by dividing the length of the walkway by the time taken by the participant at his natural pace from one end of the walkway to another. The maximum gait speed $(\mathrm{m} / \mathrm{s})$ was obtained by dividing the length of the walkway by the time taken by the participant to walk at his maximal safe speed from one end of the walkway to the other. The cadence (step/minute) was determined by dividing the number of steps obtained on the walkway by the time taken to cover the walkway. Gait parameters and societal integration were measured pre and post training.

\section{Data analysis}

Data collected was analyzed with SPSS microcomputer software version 20. Means and Standard deviation were computed for all variables. Statistical analysis using the independent t-test was performed to determine significant differences between the groups and paired t-test was used to analyze the outcomes within the two groups. The level of significance was set at $p<0.05$.

\section{Results}

\section{Demographic data of the subjects}

The demographic data of the 35 participants who completed the study are shown in Table 1. The group $A$ (Treadmill) consisted of 17 participants (10 males, 7 females), 64.7\% were right hemi-paretic. The group B (Bicycle ergometer) consisted of 18 participants, (8 males, 10 females), 55.6\% were right hemi-paretic. Mean age and weight of the participants were $53.77 \pm 10.95$ years and $70.86 \pm 10.67 \mathrm{~kg}$ respectively.

\section{Changes in gait parameters with treatment}

Tables 2 and 3 show the pre-treatment and post-treatment gait parameters and community integration for the subjects with 10 weeks of intervention. The pre-treatment score revealed a significant difference in stride length, gait speed and no significant difference in step length, cadence and community integration 
between the groups. The mean differences between the groups and post-treatment scores were significant in cadence between the groups.

Tables 4 and 5 show that there was a significance difference in the gait parameters and community integration within the groups except in stride length in the bicycle ergometer group $(P<0.05)$.

Table 1: Sociodemographic profile of participants

Variables
Threadmill

N (\%)
Bicycle ergometer

N (\%)

$\begin{array}{llcc}\text { Gender } & \text { Male } & 10(58.8) & 8(44.4) \\ & \text { Female } & 7(41.2) & 10(55.6) \\ \text { Educational attainment } & \text { Primary } & 3(8.6) & 2(5.5) \\ & \text { Secondary } & 6(17.1) & 3(8.6) \\ & \text { Tertiary } & 10(28.6) & 11(31.4) \\ \text { Part affected } & & & 10(55.6) \\ & \text { right } & 11(64.7) & 8(44.4)\end{array}$

\section{Key:}

N: Frequency

$\%$ : Percentage

Table 2: Pre-treatment gait parameters between groups of participants 


\begin{tabular}{lcccc}
\hline Variables & $\begin{array}{r}\text { Treadmill } \\
\text { Mean } \pm \text { SD }\end{array}$ & $\begin{array}{r}\text { Bicycle Ergometer } \\
\text { Mean } \pm \text { SD }\end{array}$ & t-value & p- \\
value & & & & \\
\hline Stride length & $77.41 \pm 25.52$ & $59.00 \pm 24.20$ & 2.187 & $0.04^{*}$ \\
Step length & $36.05 \pm 12.04$ & $28.94 \pm 14.09$ & 1.608 & 0.12 \\
Natural Gait Speed & $0.60 \pm 0.17$ & $0.42 \pm 0.27$ & 2.426 & $0.02^{*}$ \\
Maximum Gait Speed & $0.87 \pm 0.27$ & $0.59 \pm 0.38$ & 2.468 & $0.02^{*}$ \\
Cadence & $1.42 \pm 0.34$ & $1.25 \pm 0.62$ & 1.031 & 0.31 \\
CIS & $12.35 \pm 3.9$ & $9.50 \pm 4.00$ & 2.125 & 0.41 \\
\hline
\end{tabular}

Key:

CIS: Community Integration Score

Table 3: Post-treatment gait parameters of participants

\begin{tabular}{lcccc}
\hline Variables & $\begin{array}{c}\text { Treadmill } \\
\text { Mean } \pm \text { SD }\end{array}$ & $\begin{array}{c}\text { Bicycle ergometer } \\
\text { Mean } \pm \text { SD }\end{array}$ & t-value & p-value \\
& $6.00 \pm 5.93$ & $2.83 \pm 6.41$ & 1.519 & 0.13 \\
Stride length & $4.11 \pm 2.93$ & $2.33 \pm 2.43$ & 1.955 & 0.06 \\
Step length & $0.19 \pm 0.09$ & $0.11 \pm 0.16$ & 1.731 & 0.10 \\
Natural gait speed & $0.18 \pm 0.24$ & $0.09 \pm 0.14$ & 1.413 & 0.17 \\
Maximum gait speed & $0.20 \pm 0.18$ & $0.83 \pm 0.15$ & 2.088 & $0.04 *$ \\
Cadence & $4.00 \pm 2.98$ & $3.89 \pm 2.40$ & 0.121 & 0.90 \\
CIS & & & & \\
\hline
\end{tabular}

Key:

CIS: Community Integration Score

Table 4: Pre-treatment and post-treatment gait parameters in the treadmill group 
Parameters

Mean \pm SD

Mean \pm SD

t-value

p-value

Pre-intervention Post-intervention

Stride Length (cm) $77.41 \pm 25.52$

$83.41 \pm 21.49$

$-4.174$

$0.001 *$

Step Length (cm) $\quad 36.05 \pm 12.04$

$40.18 \pm 11.35$

$-5.786$

0.00*

NGS (m/s)

$0.60 \pm 0.17$

$0.79 \pm 0.17$

$-9.030$

$0.00 *$

MGS (m/s)

$0.87 \pm 0.27$

$1.05 \pm 0.22$

$-3.110$

$0.007 *$

Cadence (step/min) $1.42 \pm 0.34$

$1.62 \pm 0.32$

$-4.632$

0.00*

CIS

$12.35 \pm 3.94$

$16.35 \pm 3.24$

$-5.536$

$0.00 *$

Key:

CIS: Community Integration Score

NGS: Natural gait speed

MGS: Maximum gait speed

Table 5: Pre-treatment and post-treatment gait parameters in the bicycle ergometry group

Parameters

Mean \pm SD

Pre-intervention
Mean \pm SD

t-value p-value

\section{Post-intervention}

$\begin{array}{lcccc}\text { Stride Length }(\mathrm{cm}) & 59.00 \pm 24.20 & 61.83 \pm 22.71 & -1.875 & 0.078 \\ \text { Step Length }(\mathrm{cm}) & 28.94 \pm 14.09 & 31.28 \pm 13.83 & -4.082 & 0.00 \text { * }^{*} \\ \text { NGS }(\mathrm{m} / \mathrm{s}) & 0.42 \pm 0.27 & 0.53 \pm 0.27 & -2.942 & 0.009^{*} \\ \text { MGS (m/s) } & 0.59 \pm 0.38 & 0.68 \pm 0.43 & -2.631 & 0.018^{*} \\ \text { Cadence (step/min) } & 1.25 \pm 0.62 & 1.33 \pm 0.63 & -2.410 & 0.028^{*} \\ \text { CIS } & 9.50 \pm 4.00 & 13.39 \pm 3.43 & -6.880 & 0.00^{*}\end{array}$

\section{Key:}


CIS: Community Integration Score

NGS: Natural gait speed

MGS: Maximum gait speed

\section{Discussion}

This study compared the effect of open-chain kinematics (bicycle ergometer) and closed-chain kinematics (treadmill) on improving walking proficiency in post-stroke individuals in order to provide foundation for developing evidence-based gait training in enhancing functional independence and promoting societal integration after stroke. The almost equal number of males (18) and females (17) in this study shows that there is no sex disparity in the epidemiology of stroke. This corroborates the results of previous studies in Nigeria which have shown no gender variability in the occurrence of stroke [17-20]. The mean age ( $53.77 \pm 10.45$ years) of the participants corroborates the report that stroke is more common among individuals who are above 45 years old [21,22]. The result that the right side of the body was more affected than the left side shows that the left hemisphere is more predisposed to stroke than the right hemisphere of the brain. This may not be unconnected with the fact that most people have their dominant cerebral hemisphere at the left and there is more functional orientation from the left cerebral hemisphere [23].

Ambulatory parameters including reduced stride length, speed length (natural and maximal), step length and cadence have been identified as major challenge among stroke patients [11]. This is sequel to motor and sometimes sensory deficit that is associated with stroke and precipitates functional dependency after stroke [24]. Any strategy that will improve ambulatory proficiency in stroke survivors will reduce both primary and secondary burden of stroke and improve productivity [5]. Hence, the results that both an open-chain and a closed-chain kinematics are effective in re-educating ambulation and re-gaining these spatio-temporal gait parameters after stroke provide a way out of the inherent dependency in activity of daily living and disability which are common occurrences with stroke [25]. This calls for the integration of kinematics training into stroke rehabilitation as early as ambulatory performance is to be re-educated. Hence, structuring and integrating kinematics training into stroke patients' treatment regimen may effectively improve their functional capability and functional independence performance after stroke.

Findings in the present study revealed that those in the closed-chain kinematics treatment performed significantly better than the open-chain group in cadence when they are comparable in their improvement in other gait parameters, thus suggesting that closed-chain kinematics has a relatively better advantage in improving gait functional performance after stroke. This better improvement may be explained by the fact that a closed-chain kinematics is a weight bearing exercise which is likely to impact positively on the joint proprioception through the process of joint approximation [26]. Increased proprioception will increase confidence in ambulation as there will be increase in joint positional sense [27]. In addition to improved spatial orientation, weight-bearing exercises as is provided by closed-chain kinematics impact positively on muscle strength and coordination [28]. This is in agreement with previous report that 
maintenance of postural balance and gait includes a sensory process involving the articular mechanoreceptors, vestibular system and visual system [29]. Sensorimotor information is then processed in the central nervous system producing a motor response involving various muscle groups such as those around the ankle, thigh and trunk. This improvement could also be attributed to increase in muscle strength and enhanced coordination between both sides of the body. This is supported by other studies. An appropriate motor response for postural balance control and gait training requires an intact neuromuscular system and sufficient muscle strength to return the center of mass within the base of support [30]. The better improvement that occurred in the treadmill group may be due to the stepping practice. Stepping practice strengthened and stabilized the neural network which was involved in producing this pattern [28]. This is in agreement with other reports that treadmill intervention offered repeated opportunities to improve gait and build muscle strength in the lower limbs and stimulate neuronal connections that are involved in generation of independent balanced walking [31, 32].

Although both groups improved significantly in their six minutes-walk performance after intervention, the closed chain group performed relatively better. This may not be unconnected with the fact that the treadmill trains gait and gait-speed and improves ambulatory performance [33]. This supported the findings of Kubo and Ulrich [34] that treadmill training improves the spatio-temporal parameters of gait and produces more normal temporal pattern. It has been shown that the treadmill is considered as a moving surface, so, the post-stroke participants needed to spend more time with both feet on the surface during the walking cycle than when they walked over ground [35]. Furthermore, gait stability, muscle function, aerobic fitness and balance will improve more in participants receiving treadmill training and also community re-integration will be higher [36]. This shows that treadmill training is most effective in achieving spatio-temporal gait parameters in this study and hence, adds further support to a growing body of evidence that treadmill exercise training gives better improvement in gait training in patients with cerebrovascular disease $[37,38]$.

The results of this study also show that both open-chain and closed-chain kinematics significantly improved the community re-integration in individuals recovering from stroke, and this may be associated with their improvement in gait performances. When an individual can walk better, he/she is likely to manipulate his/her environment better for community functioning [39]. If individuals can function in their community, they will be better re-integrated and contribute to the community. Community re-integration has been shown to be positive outcomes such as motor function after stroke and it is positively associated with improved quality of life [40], subjective well-being and life satisfaction after stroke [41]. Improvement in walking proficiency has been reported to result in significant improvements in the mobility, physical independence performance, works and productivity, and leisure activities after stroke [42].

The better improvement in the community integration of the closed-chain group can be explained by their better performance in both walking independence performance and gait parameters such as cadence. Earlier studies have demonstrated that improvement in mobility will improve every aspect of functional independence performance and quality of life after stroke [41, 42]. Physiotherapist should direct their 
rehabilitation efforts at improving quality gait functioning for better societal integration and participation in stroke patients and the close-chain kinematics should be a preferred approach to achieving these.

\section{Conclusions}

Open-chain kinematics and closed-chain kinematics effectively improve walking proficiency and societal integration of post-stroke individuals, although closed-chain gave a better improvement. This calls for the integration of kinematics training into stroke rehabilitation as early as ambulatory performance is to be re-educated.

We therefore recommend that future trials should focus more upon longer duration exercise programs for enhancing the walking proficiency of post-stroke individuals. Larger randomized studies are needed to generate evidence-based guidelines for the safe and effective exercise of post-stroke individuals across the spectrum of deficit profiles and recovery phases. It is important to include open-chain kinematics or closed-chain kinematics (bicycle ergometry and/or treadmill training) in the management of stroke. Further studies are required to investigate the mechanisms underlying the observed improvement in gait parameters. One limitation of the present study is the small sample size. Also, the participants were recruited from tertiary institutions, thereby, limiting the generalizability of the results.

\section{References}

1. Belda-Lois, J. M., Horno, S. M., Bermejo, B. J., \& Moreno, J. C. (2011). Rehabilitation of gait after stroke: a review towards a top-down approach. Journal of NeuroEngineering and Rehabilitation; $8(66), 1-19$

2. Barthélemy, M. J., Grey, J. B., Nielsen, L., \& Bouyer, M. (2011). Involvement of the corticospinal tract in the control of human gait. Progress in Brain Research, 192, 181-197

3. Gwin, J. L., Gramann, K., Makeig, S., \& Ferris, P. P. (2011). Electrocortical activity is coupled to gait cycle phase during treadmill walking. Neurolmage-Journal;; 54(2), 1289-1296

4. Presacco, R., Goodman, L., Forrester, J. L., \& Contreras, V. (2011). Neural decoding of treadmill walking from noninvasive electroencephalographic signals. Journal of Neurophysiology; 106(4), 1875-1887

5. Takatori, K. Y., Okada, K., Shomoto, K., Ikuno, K., Nagino, K., \& Tokuhisa, P. (2012). Effect of a cognitive task during obstacle crossing in hemiparetic stroke patients. Physiotherapy Theory and Practice; 28(4), 292-298

6. Vasudevan, V. L., \& Bastian, A. J. (2010). Split-belt treadmill adaptation shows different functional networks for fast and slow human walking. Journal of Neurophysiology; 103(1), 183-191

7. Balaban, B. (2010). Gait Disturbances in patients with stroke. Journal of Neurology, 56(8), 642-655

8. Swigchem, H. J., Duijnhoven, J., Boer, A. C., Geurts, V., \& Weerdesteyn, V. (2013). Deficits in motor response to avoid sudden obstacles during gait in functional walkers poststroke. Neurorehabilitation and Neural Repair; 27(3), 230-239

Page $12 / 15$ 
9. Heeren, A., Van, M. N., Ooijen, A. C., \& Geurts, H. (2013). Step by step: a proof of concept study of CMill gait adaptability training in the chronic phase after stroke. Journal of Rehabilitation Medicine, 45(7), 616-622

10. Torres, M., Zumbansen, A., \& Sparks, G. D. (2011). Recent advances in the treatment of post-stroke aphasia. Stroke, 57(20), 234-244

11. Juan, M. B., \& Lois, T. (2011). Rehabilitation of gait after stroke: A review towards a top down approach. Journal of Neurology Engineering and Rehabilitation, 8, 66-68

12. Janice, J. E., \& Pei, F. T. (2012). Gait training strategies to optimize walking ability in people with stroke: A synthesis of the evidence. Expert Review of Neurotherapeutics, 7(10), 1417-1436

13. Carr, J. H., \& Shepherd, R. B. (2003). Stroke Rehabilitation- Guidelines for exercise and Training to optimize motor skill, (2nd edition) Lippincot Williams and Wilkins, New York.

14. Hill, K., Ellis, P., \& Bernhardt, J. (2010). Balance and mobility outcomes for stroke patients: a comprehensive Audit. Australian Journal of Physiotherapy; 43, 173-180

15. Eid, T., Palomba, M., De-Lanerolle, N. C., \& Nagelhus, E. A. (2014). The central role of aquaporins in the pathophysiology of ischemic stroke. Stroke, 45, 267-269

16. Willer, B., Ottenbacher, K. J., \& Coad, M. L. (1994). The community integration questionnaire. A comparative examination. American Journal of Physical Medical Rehabilitation; 73(2), 103-111

17. Ogun, S. A., Ojini, F. I., Ogungbo, B., Kolapo, K. O., \& Danesi, M. A. (2005). Stroke in South West Nigeria. A 10 year review. Journal of Stroke; 36, 1120-1122

18. Akinpelu, A. O., \& Gbiri, C. A. (2009). Quality of life of Stroke Survivors and Apparently Healthy Individuals in South-western Nigeria. Physiotherapy Theory and Practice; 25, 14-20

19. Owolabi, M. O. (2011). Impact of stroke on health-related quality of life in diverse cultures: the BerlinIbadan multicenter international study. Health and Quality of Life Outcomes; 20, 29-81

20. Gbiri, C. A., Akinpelu, A. O., \& Odole, A. C. (2014). Prevalence, Pattern and Impact of Depression on Quality of Life of Stroke Survivors. International Journal of Psychiatry in Clinical Practice; 14, 198203

21. Danesi, M., Okubadejo, N., \& Ojini, F. (2007). Prevalence of stroke in an urban, mixed-income community in Lagos, Nigeria. Journal of stroke neuroepidemiology; 28, 216-223

22. Olawale, O. A., Jaja, S. I., Anigbogu, C. N., Appiah-Kubi, K., \& Jones-Okai, D. (2009). Effects of two exercise training techniques on walking function in adult patients with stroke. Journal of Ghanaian University; 40(10), 357-360

23. Smulders, K., Van, R., Swigchem, B. J., De Swart, A. C., Geurts, H., \& Weerdesteyn, A. (2012). Community-dwelling people with chronic stroke need disproportionate attention while walking and negotiating obstacles. Gait and Posture; 36(1), 127-132

24. Roger, V. L., Go, A. S., Lloyd-Jones, D. M., \& Benjamin, E. J. (2012). Heart disease and stroke statistics2012 update: a report from the American Heart Association. Circulation; 125(1): 2-220. 
25. Lin, J. H., Hsu, M. J., Wu, H. C., \& Hsieh, C. L. (2010). Psychometric comparisons of 3 functional ambulation measures for patients with stroke. Stroke; 41(9), 2021-2025

26. Mosley, P. J., \& De-Souza, S. P. (2005). Measurement of joint spectral intensity. Physical Review; 56(9), 36-39

27. Wade, D. T., Wood, V. A., \& Heller, A. (2010). Walking after stroke: measurement and recovery over the first three months. Scandivian Journal of Rehabilitation Medicine; 19, 25-30

28. Visintin, M., \& Barbeau, H. (2009). The effects of body weight support on the locomotor pattern of spastic paretic patients. Canadian Journal of Neurology; 16, 315-325

29. Guskiewicz, K., Prentice, W., \& Voight, M. (2002). Impaired postural stability: regaining balance in techniques in musculoskeletal rehabilitation. Journal of Rehabilitation Medicine; 8(24), 125-150

30. Karimi, N., Ebrahimi, I., \& Kahrizi, S. (2008). Evaluation of postural balance using the Biodex balance system in subjects with and without low back pain. Pakistan Journal of Medical Sciences; 6, 45-52

31. Ulrich, D. A., Ulrich, B. D., \& Angulo-Kinzler, R. M. (2001). Treadmill training of infants with Down syndrome: evidence-based developmental outcomes. Pediatrics; 108, 84-91

32. Dal, U., Erdogan, T., \& Resitoglu, B. (2010). Determination of preferred walking speed on treadmill may lead to high oxygen cost on treadmill walking. Gait Posture; 31, 366-399

33. Hesse, S., Bertelt, C., Schaffrin, A., Malezic, M., \& Mauritz, K. H. (2011). Restoration of gait in nonambulatory hemiparetic patients by treadmill training with partial body-weight support. Archives of Physical Medical Rehabilitation; 75, 1087-1093

34. Kubo, M., \& Ulrich, B. (2006). Coordination of pelvis-HAT (head, arms and trunk) in anterior-posterior and medio-lateral directions during treadmill gait in pre adolescents with/without Down syndrome. Gait Posture; 23, 512-518

35. Matsuno, V. M., Camargo, M. R., \& Palma, G. C. (2010). Analysis of partial body weight support during treadmill and over ground walking of children with CP. Brazillian Journal of Physical Therapy, 14(5), $23-25$

36. Tulchin, K., Orendurff, M., \& Karol, L. (2010). A comparison of multisegment foot kinematics during level over ground and treadmill walking. Gait Posture; 2, 104-108

37. Macko, R. F., Ivey, F. M., Forrester, L. W., Hanley, D., Sorkin, J. D., Katzel, L. I., et al. (2005). Treadmill Exercise Rehabilitation Improves Ambulatory Function and Cardiovascular Fitness in Patients With Chronic Stroke: A Randomized Controlled Trial. Stroke;36: 2206-2211.

38. Pang, M. Y. C., Eng, J. J., Dawson, A. S., \& Gylfadottir, S. (2006). The use of aerobic exercise training in improving aerobic capacity in individuals with stroke a meta-analysis. Clinical Rehabilitation; 20, 97-111

39. Woodman, P., Afsane, R., Pereira, C., \& Jones, F. (2014). Social participation post stroke: a metaethnographic review of the experiences and views of community-dwelling stroke survivors. Archives of Physical and Medical Rehabilitation; 69, 583-590 
40. Kwok, J. H., Pan, R., \& Song, X. (2011). The influence of participation on health-related quality of life in stroke patients. Disability and Rehabilitation; 33(22), 1990-1996

41. Vestling, M., Tufvesson, B., \& Iwarsson, C. (2003). Indicators for return to work after stroke and the importance of work for subjective well-being and life satisfaction. Journal of Rehabilitation Medicine; 35(3), 127-131

42. Berge, C., Andrew, S., \& Allen, J. L. (2013). Long term training modifies the modular structure and organization of walking balance control. Journal of Neurology; 114(6), 3359-3373

\section{Declarations}

\section{Funding}

Not applicable.

\section{Authors' contributions}

All authors contributed to the study conception and design. Material preparation, data collection and analysis were performed by OCN, CAOG and OAO. The first draft preparation and reviewing of the manuscript were performed by UGM and CAA. All authors commented on the previous versions of the manuscript and approved the final manuscript.

\section{Acknowledgements}

Not applicable

Declaration of competing interests

The authors declare no competing interests. 